\title{
MODELLING, ANALYSIS AND SIMULATION OF A NOVEL AUTOMATED MANUAL TRANSMISSION WITH GEARSHIFT ASSISTANT MECHANISM
}

\author{
Zhiqiang Sun ${ }^{1,2)}$, Bingzhao Gao ${ }^{3)}$, Jiaqi Jin $^{1)}$ and Kazushi Sanada ${ }^{2 *}$ \\ ${ }^{1)}$ School of Mechanical Engineering, Shenyang University of Technology, Shenyang 110000, China \\ ${ }^{2}$ Department of Mechanical Engineering, Yokohama National University, Yokohama 240-8501, Japan \\ ${ }^{3)}$ State Key Laboratory of Automotive Simulation and Control, Jilin University, Changchun 130025, China
}

(Received 19 July 2018; Revised 14 December 2018; Accepted 24 January 2019)

\begin{abstract}
To eliminate or reduce torque interruption and driveline jerk for traditional automated manual transmission (AMT), AMT with a gearshift assistant mechanism (GAM) is originally proposed in this paper. The GAM consists of a torque complementary motor and an epicyclic mechanism with a synchronizing clutch. During gear upshift, the electrical motor provides complementary torque to primary (output) shaft after synchronizer discharges, then the synchronizing clutch will work to synchronize primary shaft with anticipated gear. The lockup of the synchronizing clutch will ensure the synchronization of primary shaft and anticipated gear. After finishing synchronizing, synchronizer will lock up the anticipated gear and engine recovers torque supply to finish gearshift. Based on the mathematical model of the proposed transmission, its detailed structure, kinematic character and dynamic behavior are discussed. Controllers are designed to achieve presumed gearshift performance, and simulation results show its effectiveness. Problems may be encountered in engineering application and possible application on electrical vehicle (EV) of the proposed transmission are also discussed. Finally, this paper summarized the merits and further research targets of the proposed transmission, which is a promising structure to achieve swift and smooth gearshift.
\end{abstract}

KEY WORDS : Automated manual transmission, Power-shift transmission, Gearshift assistant mechanism, Shift control

\section{NOMENCLATURE}

$\omega_{\mathrm{e}} \quad$ : engine output shaft angular velocity, $\mathrm{rad} / \mathrm{s}$

$\omega_{\mathrm{p}} \quad$ : primary shaft angular velocity, $\mathrm{rad} / \mathrm{s}$

$\tau_{\mathrm{i}} \quad$ : transmission gear ratio in $i$ th gear, -

$\omega_{\text {anti }}:$ anticipated gear angular velocity, $\mathrm{rad} / \mathrm{s}$

$\omega_{\text {scl }_{\mathrm{e}}}$ : angular velocity of synchronizing clutch pad on the engine side, $\mathrm{rad} / \mathrm{s}$

$\omega_{\text {scl }_{\mathrm{p}}}$ : angular velocity of synchronizing clutch pad on the primaryshaft side, $\mathrm{rad} / \mathrm{s}$

$\Delta \omega_{\mathrm{ap}}$ : angular velocity difference of primary shaft and anticipated gear, $\mathrm{rad} / \mathrm{s}$

$\Delta \omega_{\text {scl }}$ : angular velocity difference of synchronizing clutch pads, $\mathrm{rad} / \mathrm{s}$

$\tau_{\text {es }}$ : gear ratio of engine output shaft and secondary shaft, -

$\tau_{\text {epi }} \quad$ : gear ratio of epicyclic mechanism in $i$ th gear, -

$\tau_{\mathrm{c}} \quad$ : gear ratio of complementary gear, -

$\tau_{\text {spi }}$ : gear ratio of anticipated gear pair in ith gear, -

$\tau_{\mathrm{df}} \quad$ : gear ratio of differential gear, -

$I_{\mathrm{e}} \quad$ : moment of inertia of engine, $\mathrm{kgm}^{2}$

$I_{\mathrm{mc}} \quad$ : moment of inertia of main clutch, $\mathrm{kgm}^{2}$

*Corresponding author. e-mail: sanada-kazushi-sn@ynu.ac.jp
$I_{\mathrm{s}} \quad:$ moment of inertia of secondary shaft, $\mathrm{kgm}^{2}$

$I_{\mathrm{v}} \quad:$ moment of inertia of driveline, $\mathrm{kgm}^{2}$

$C_{\mathrm{s}} \quad$ : damping coefficient of engine, $\mathrm{Nm}(\mathrm{rad} / \mathrm{s})$

$C_{\mathrm{mc}}$ : damping coefficient of main clutch, $\mathrm{Nm}(\mathrm{rad} / \mathrm{s})$

$C_{\mathrm{s}} \quad$ : damping coefficient of secondary shaft, $\mathrm{Nm}(\mathrm{rad} / \mathrm{s})$

$C_{\mathrm{v}} \quad$ : damping coefficient of driveline, $\mathrm{Nm}(\mathrm{rad} / \mathrm{s})$

$T_{\mathrm{e}} \quad$ : engine torque, $\mathrm{Nm}$

$T_{\text {scl }} \quad$ : synchronizing clutch torque, $\mathrm{Nm}$

$T_{\mathrm{r}} \quad$ : resistent torque, $\mathrm{Nm}$

$T_{\mathrm{w}} \quad$ : tire rolling resistant torque, $\mathrm{Nm}$

$R_{\mathrm{w}} \quad:$ tire radius, $\mathrm{m}$

$C_{\mathrm{A}} \quad$ : constant coefficient, -

$m$ : vehicle mass, $\mathrm{kg}$

$g \quad:$ gravitational acceleration, $\mathrm{m} / \mathrm{s}^{2}$

$\theta \quad$ : road inclination, ${ }^{\circ}$

$\dot{\omega}_{\mathrm{p}} \quad$ : angular acceleration of primary shaft, $\mathrm{rad} / \mathrm{s}^{2}$

$\dot{\omega}_{\mathrm{p}_{\mathrm{ref}}}$ : reference angular acceleration of primary shaft, rad/ $\mathrm{s}^{2}$

$\dot{\omega}_{\mathrm{p} 0}:$ measured angular acceleration of primary shaft, rad/ $\mathrm{s}^{2}$

$\dot{\omega}_{\mathrm{e}} \quad$ : angular acceleration of engine output shaft, $\mathrm{rad} / \mathrm{s}^{2}$

$\dot{\omega}_{\text {scl }_{\mathrm{p}}}$ : angular acceleration of synchronizing clutch pad on the priamary shaft side, $\mathrm{rad} / \mathrm{s}^{2}$

$\dot{\omega}_{\text {scl }_{\mathrm{e}}}$ : angular acceleration of synchronizing clutch pad on 
the engine side, $\mathrm{rad} / \mathrm{s}^{2}$

$\Delta \dot{\omega}_{\mathrm{sc}_{\mathrm{e}}}$ : estimated angular acceleration of synchronizing clutch pad on the engine side, $\mathrm{rad} / \mathrm{s}$

$\Delta \dot{\omega}_{\text {scl }}:$ angular acceleration difference of synchronizing clutch pads, $\mathrm{rad} / \mathrm{s}^{2}$

$\Delta \omega_{\text {sclref }}$ : reference angular speed difference of synchronizing clutch pads, $\mathrm{rad} / \mathrm{s}$

$\Delta \omega_{\text {sclo }}:$ measured angular speed difference of synchronizing clutch pads, $\mathrm{rad} / \mathrm{s}$

$\hat{T}_{\mathrm{e}} \quad$ : estimated engine torque, $\mathrm{Nm}$

$\hat{T}_{\mathrm{r}} \quad$ : estimated resistant torque, $\mathrm{Nm}$

$\dot{T}_{\text {scl }}$ : change of synchronizing clutch torque, $\mathrm{Nm} / \mathrm{s}$

$\dot{T}_{\mathrm{m}}$ : change of motor torque, $\mathrm{Nm} / \mathrm{s}$

$\hat{T}_{\mathrm{m}}$ : estimated motor torque, $\mathrm{Nm}$

$T_{\text {out }}$ : output torque, $\mathrm{Nm}$

$t_{\mathrm{f}} \quad$ : gear shift finish time, $\mathrm{s}$

\section{SUBSCRIPTS}

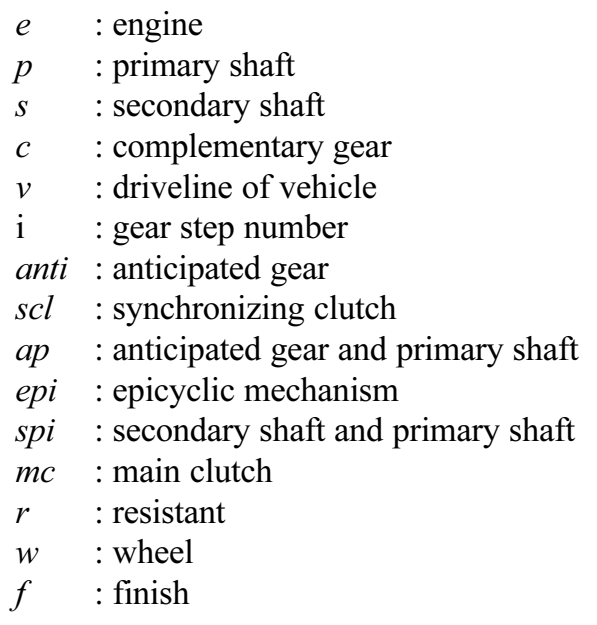

\section{INTRODUCTION}

As the surging demand of the general publics for more environmental friendly and more comfort vehicle, transmission has been developed to be swifter and smoother in recent years (Maruyama et al., 2017). For electric vehicle (EV), due to its low efficiency and the current low energy-storage capacity of electric batteries, new transmission structures have been contrived, such as multi-speed transmission (MST) proposed in Roozegar and Angeles (2018) and Roozegar et al. (2017), and the inverse automated manual transmission (I-AMT) proposed in Gao et al. (2015). For inner combustion engine vehicle, to achieve better performance than traditional manual transmission (MT), generally four types of transmissions have been developed: continuously variable transmission (CVT), dual clutch transmission (DCT), automatic transmission (AT) and automated manual transmission (AMT).

Generally, MT is constituted of a main clutch and a multi-speed gearbox. As concluded in Galvagno et al.
(2011), MT shows over $10 \%$ higher overall efficiency than AT and CVT. Due to such a reason, automated manual transmissions (AMT) are invented based on MT structure to achieve better gear shift performance. AMT are MT equipped with controllable actuators and control units to automatically achieve gear shift. AMT has the advantages of lower cost, lower weight, and easy to put into production. As discussed in Lin et al. (2014), AMT is also considered a promising transmission structure for electric vehicle.

The gearshift problems of AMT could be concluded as follow:

(1) Torque interruption (torque gap) while main clutch or synchronizer is disengaged.

(2) Driveline jerk while main clutch slipping and lockup.

To fill the torque gap, several methods have been proposed: 1. The torque gap filler (TGF) designed by Sorniotti (2009) consists of an epicyclic gear-set transmits engine torque to AMT secondary shaft during gearshift. 2 . Transmission with a flywheel to store energy for torque interruption as demonstrated (Galvagno et al., 2009). 3. Assist clutch (ACL) proposed in Galvagno et al. (2011), ACL replaces the fifth gear synchronizer and allows engine torque from secondary shaft to be transmitted through ACL to the primary shaft during gearshift. 4. Torque complementary motor provides complementary torque for Suzuki auto gear shift (AGS) AMT to fill the torque gap during torque interruption as demonstrated (Shoji et al., 2017).

To reduce the driveline jerk while main clutch slipping and lockup, different control strategies are developed for steady and swift clutch engagement, such as lurch avoidance control (Dolcini et al., 2008) data-driven predictive control (Lu et al., 2011), observer-based clutch disengagement control (Gao et al., 2011a), model predictive control (Gao et al., 2011b, 2013b), improved optimal control (Gao et al., 2013a), etc.

To simultaneously fix the two gearshift problems, and achieve swifter and smoother gearshift, AMT with a GAM is originally proposed in this paper. The GAM consists of a torque complementary motor and an epicyclic mechanism with a synchronizing clutch. During gear upshift, electrical motor provides complementary torque to primary shaft after the synchronizer discharge, then the synchronizing clutch pads start to engage. The lockup of the synchronizing clutch will ensure the synchronization of primary shaft and anticipated gear. After the synchronization process, synchronizer will lock up the anticipated gear, and engine recovers torque supply to finish gearshift. The main clutch of the proposed transmission will only be used in vehicle start-up process.

Compared with the traditional AMT, the proposed structure can directly recover engine torque supply after synchronizer locks up instead of by engaging the main clutch. The GAM with identical gear steps as the transmission accelerates gearshift speed and consequently 
shorten the torque interruption duration. The torque interruption is promising to be fixed or to be reduced by the relative short gear shift time and the torque complementary motor. Meanwhile, the new structure empowers the equipped automotive to achieve several hybrid vehicle functions, such as launch by motor, energy regeneration, etc. It also worth noting that it will only take two speed sensors for the proposed transmission to swiftly finish gearshift.

Details of the proposed structure will be introduced in the next section. The analysis will focus on the system architecture, the system dynamics behavior and the control algorithm design. Finally, some simulation results are reported to validate the effectiveness of the proposed architecture.

\section{DESCRIPTION OF PROPOSED NEW ARCHITECTURE}

The new architecture (see Figure 1) consists of a traditional automated manual transmission, a complementary electrical motor, and a set of epicyclic mechanism with a friction clutch. For the sake of simplicity, only two of the total five gear steps of the traditional AMT are indicated in Figure 1. The red gears are fixed on the shaft and green gears are free to the shaft. The blue synchronizer ( 5 in Figure 1) is randomly romanced and hyalinized for a better presentation of its inside structures. The complementary electrical motor simultaneously provides torque to the primary shaft through a pair of complementary gears and to the secondary shaft through the epicyclic mechanism. The epicyclic mechanism consists of three epicyclic gearsets with clutches and brakes. By coordinating the state of the brakes and the clutches of sun gears, ring gears and planet

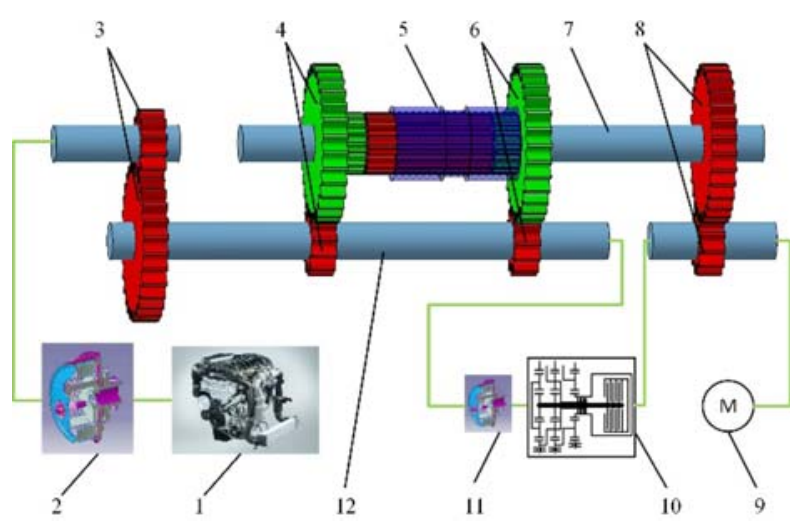

Figure 1. Simplified layout of AMT with a gearshift assistant mechanism: 1 - Inner combustion engine; 2 Main clutch; 3 - Gear pair connect the engine output shaft and the secondary shaft; 4 - Anticipated gear pair; 5 Synchronizer; 6 - Present gear pair; 7 - Primary shaft (output shaft); 8 - Complementary gears; 9 - Torque complementary motor; 10 - epicyclic mechanism; 11 The synchronizing clutch; 12 - The secondary shaft. carriers, the epicyclic mechanism provides six or more (when necessary) different gear ratios. The details of the epicyclic mechanism is neglected here, since a mechanism that output expected gear ratios is essential here, the epicyclic mechanism could be replaced by any lower cost mechanism with similar function. In the case of AMT with five gear steps, five gear steps would be required for the epicyclic mechanism to achieve the function of both upshift and downshift. the gear step optimization of GAM will be carried out in further research to discuss the implementation costs variation with respect to synchronizing time and friction loss. The friction clutch (see 23 in Figure 1) connects the output part of the epicyclic mechanism with the secondary shaft of the gearbox is the synchronizing clutch. The integrated system is electrically driven by a control unit that coordinate the controls of the engine, the electrical motor, the synchronizing clutch, the gear selection of the gear box and the gear selection of the epicyclic mechanism.

The internal combustion engine is coupled with the secondary shaft by the main clutch and a pair of gears; synchronizer (see 4 in Figure 1) on the primary shaft (which is also the output shaft) locks up the anticipated gear with the primary shaft by its dog teeth; the gear ratios of the proposed epicyclic mechanism are designed with respect to the gear ratios of the transmission and that of the complementary gear pair, which enable the synchronizing clutch ensure the synchronization of the primary shaft and the anticipated gear; the proposed complementary electrical motor directly provides complementary torque to the primary shaft through the complementary gear pair.

In general, the gearshift of the proposed structure is accomplished by the following steps: 1. the gear ratio selection of the epicyclic mechanism; 2. discharge of the synchronizer dog teeth; 3. complementary motor provide complementary torque and synchronizing clutch start to engage; 4. synchronizer dog teeth lock up the anticipated gear after the synchronizing clutch locks up. 5. engine recover the torque supply.

\section{KINEMATIC ANALYSIS}

The driveline can be descried only by two angular speeds: engine speed $\omega_{\mathrm{e}}$ and primary shaft speed $\omega_{\mathrm{p}}$. When the vehicle normally cruises, the rotational system could be described as follow:

$\omega_{\mathrm{p}}=\frac{\omega_{\mathrm{e}}}{\tau_{\mathrm{i}}}$

where $\tau_{\mathrm{i}}$ is the gear ratio of the $i$ th gear.

During gear upshift, when the engaged synchronizer dog teeth discharged from the primary shaft, the synchronizing clutch pads start engaging. Analyzing the relationship between $\omega_{\mathrm{e}}$ and $\omega_{\mathrm{p}}$, the status of the anticipated gear and the synchronizing clutch pads at this moment, it holds: 


$$
\begin{aligned}
& \omega_{\mathrm{p}}=\frac{\omega_{\mathrm{e}}}{\tau_{\mathrm{i}}} \\
& \omega_{\text {anti }}=\frac{\omega_{\mathrm{e}}}{\tau_{\mathrm{i}+1}} \\
& \omega_{\text {scl }_{\mathrm{e}}}=\frac{\omega_{\mathrm{e}}}{\tau_{\mathrm{es}}} \\
& \omega_{\mathrm{scl}_{\mathrm{p}}}=\omega_{\mathrm{p}} \tau_{\mathrm{epi}} \tau_{\mathrm{c}}
\end{aligned}
$$

where $\omega_{\text {anti }}, \omega_{\mathrm{scl}_{\mathrm{e}}}, \omega_{\mathrm{scl}_{\mathrm{p}}}$ are respectively the angular velocity of the anticipated gear, of the synchronizing clutch pad on the engine side and other one on the primary shaft side; $\tau_{\mathrm{es}}$ is the gear ratio from the engine output shaft to the secondary shaft; $\tau_{\text {epi }}$ is the $i$ th gear ratio of epicyclic mechanism; $\tau_{\mathrm{c}}$ is the gear ratio of the complementary gear.

Subtract Equation (2) from Equation (3), the angular velocity difference between primary shaft and anticipated gear $\Delta \omega_{\text {ap }}$ could be described as:

$$
\Delta \omega_{\mathrm{ap}}=\omega_{\mathrm{anti}}-\omega_{\mathrm{p}}=\frac{\omega_{\mathrm{e}}}{\tau_{\mathrm{i}+1}}-\omega_{\mathrm{p}}
$$

As the design principle of epicyclic mechanism mentioned in Section 2, $\tau_{\text {epi }}$ will hold:

$$
\tau_{\mathrm{epi}} \tau_{\mathrm{c}}=\tau_{\mathrm{spi}}=\frac{\tau_{\mathrm{i}+1}}{\tau_{\mathrm{es}}}=\tau(i), i=1,2,3,4
$$

where $\tau_{\text {spi }}$ is the gear ratio of the anticipated gear pair from secondary shaft to primary shaft in $i$ th gear; $\tau$ indicates the vector of gear ratio values.

Letting $\Delta \omega_{\mathrm{scl}}=\omega_{\mathrm{scl}_{\mathrm{e}}}-\omega_{\mathrm{scl}_{\mathrm{p}}}$, then, Equation (6) could be rewritten as:

$\Delta \omega_{\mathrm{ap}}=\frac{\omega_{\mathrm{scl}_{\mathrm{e}}}}{\tau_{\mathrm{spi}}}-\frac{\omega_{\mathrm{scl}_{\mathrm{p}}}}{\tau_{\mathrm{epi}} \tau_{\mathrm{c}}}=\frac{1}{\tau} \Delta \omega_{\mathrm{scl}}$

According to Equation (8), $\Delta \omega_{\mathrm{scl}}$ and $\Delta \omega_{\mathrm{ap}}$ will constantly equal to zero if the synchronizing clutch locks up, which means the synchronization of the primary shaft and the anticipated gear is ensured.

To finish the gear up shift, traditional AMT takes advantage of synchronizer side surface friction to reduce $\Delta \omega_{\text {ap }}$ to zero (synchronizer work) and main clutch friction torque to reduce the angular velocity difference of the main clutch to zero (main clutch work). As comparison, the proposed structure only engages synchronizing clutch to reduce $\Delta \omega_{\text {scl }}$ to zero (synchronizing clutch work). Such a simplification on gearshift procedure is a major contribution to its swift gearshift.

\section{DYNAMIC ANALYSIS}

According to the states of the synchronizing clutch (engaged, disengaged, slipping) and of the synchronizer dog teeth (locked, unlocked), six types of degrees of freedom are assorted. Six sets of dynamic and kinematic equations will therefore be required to describe all the possible configurations.

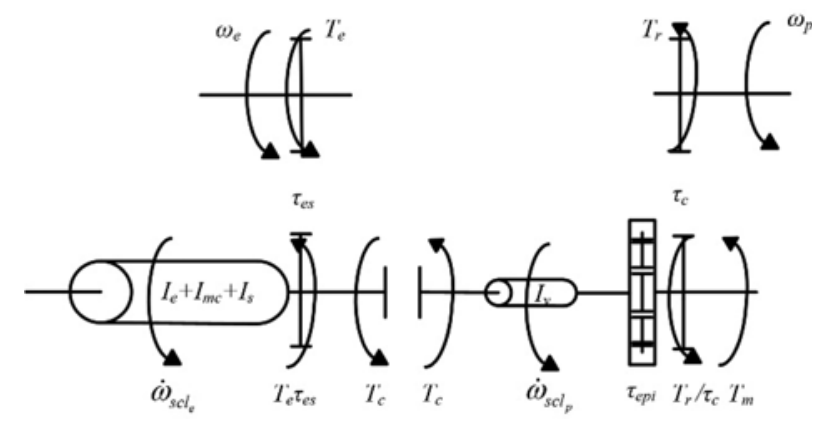

Figure 2. Free body diagram when synchronizing clutch slipping, gear disengaged.

In order to explain the dynamic behavior of the proposed automated manual transmission as simple as possible, only three cases during the upshift process are analyzed.

\subsection{Synchronizing Clutch Slipping, Gear Disengaged} After the synchronizer dog teeth discharge from the primary shaft, synchronizing clutch pads start engaging. The system, as shown in Figure 2, has two degrees of freedom: the angular velocity of the synchronizing clutch pad on the engine side $\omega_{\text {scl }_{e}}$ and of the one on the transmission primary shaft side $\omega_{\text {scl }_{1}}$. The effect of the other idle gear pairs inside of the gearbox are neglected for the sake of simplicity.

Since the main clutch is constantly engaged, the engine and the secondary shaft work as a rigid body. The dynamics of the angular velocity of secondary shaft and engine output shaft are controlled by the friction torque produced by the slipping synchronizing clutch pads and the engine torque.

Then the dynamic equation of the synchronizing clutch pad on the engine side could be derived as:

$$
\begin{aligned}
\left(I_{\mathrm{e}}+I_{\mathrm{mc}}+I_{\mathrm{s}}\right) \dot{\omega}_{\mathrm{scl}_{\mathrm{e}}}= & T_{\mathrm{e}} \tau_{\mathrm{es}}-\left(C_{\mathrm{e}}+C_{\mathrm{mc}}+C_{\mathrm{s}}\right) \frac{\omega_{\mathrm{e}}}{\tau_{\mathrm{es}}} \\
& -T_{\mathrm{scl}} \operatorname{sgn}\left(\omega_{\mathrm{scl}_{\mathrm{e}}}-\omega_{\mathrm{scl}_{\mathrm{p}}}\right)
\end{aligned}
$$

where $I_{\mathrm{e}}, I_{\mathrm{mc}}, I_{\mathrm{s}}$ are the moment of inertia of the engine, of the main clutch and of the secondary shaft; $C_{\mathrm{e}}, C_{\mathrm{mc}}, C_{\mathrm{s}}$ are the damping coefficients of the engine, of the main clutch and of the secondary shaft; $T_{\mathrm{e}}$ is the torque provided by engine; $T_{\text {scl }}$ is the friction torque produced by the proposed synchronizing clutch, and the sgn function decides the direction of $T_{\text {scl }}$ with respect to the difference of $\omega_{\text {scl }_{\mathrm{e}}}$ and $\omega_{\text {scl }}$.

Let analyze the synchronizing clutch pad on the primary shaft side, the torque complementary motor provides complementary torque to overcome the resistant torque, the friction torque produced by the synchronizing clutch and the damping effect of the rest driveline parts. Complementary torque and angular velocity of primary shaft are transferred to synchronizing clutch pads through 
the epicyclic mechanism with respect to $\tau_{\text {epi }}$ and $\tau_{\mathrm{c}}$, hence the dynamic equation of the synchronizing clutch pad on the primary shaft side could be described as:

$$
I_{\mathrm{v}} \dot{\omega}_{\mathrm{scl}_{\mathrm{p}}}=\frac{T_{\mathrm{m}}}{\tau_{\mathrm{epi}}}+T_{\mathrm{scl}} \operatorname{sgn}\left(\omega_{\mathrm{scl}_{\mathrm{e}}}-\omega_{\mathrm{scl}_{\mathrm{p}}}\right)-C_{\mathrm{v}} \omega_{\mathrm{p}} \tau_{\mathrm{epi}} \tau_{\mathrm{c}}-\frac{\tau_{\mathrm{r}}}{\tau_{\mathrm{epi}} \tau_{\mathrm{c}}}
$$

where $I_{\mathrm{v}}, C_{\mathrm{v}}$ are the moment of inertia and the damping coefficient of the rest of the driveline; $T_{\mathrm{m}}$ is the torque provided by the torque complementary motor; $T_{\mathrm{r}}$ is the resistant torque during vehicle normal cruise.

$T_{\mathrm{r}}$ consists of three parts: resistant torque form road inclination, rolling resistant torque of tires and aerodynamics drag as demonstrated (Sanada et al., 2012), it holds:

$$
T_{\mathrm{r}}=m g \sin \theta+\frac{T_{\mathrm{w}}}{\tau_{\mathrm{df}}}+\frac{c_{\mathrm{A}} R_{\mathrm{w}}^{3}}{\tau_{\mathrm{df}}^{3}} \omega_{\mathrm{p}}^{2}
$$

where $m$ is the mass of vehicle, $g$ is gravitational acceleration, $\theta$ is road inclination, $T_{\mathrm{w}}, R_{\mathrm{w}}$ are the rolling resistance torque and the radius of tire; $C_{\mathrm{A}}$ is a constant coefficient for the calculation of aerodynamic drag; $\tau_{\mathrm{df}}$ is the gear ratio of differential gear.

As the clutch pad on the primary shaft side connected with the primary shaft by epicyclic mechanism as a rigid body, the dynamics of the primary shaft could be derived as:

$$
\dot{\omega}_{\mathrm{p}}=\dot{\omega}_{\mathrm{scl}_{\mathrm{p}}} \tau_{\mathrm{epi}} \tau_{\mathrm{c}}
$$

\subsection{Synchronizing Clutch Engaged, Gear Disengaged} As the $\Delta \omega_{\text {scl }}$ reduces to around zero, the pressure of the proposed clutch will sharply increase to lock up the clutch pads, which will keep the $\Delta \omega_{\text {scl }}$ remain zero. Hence, the inner combustion engine, secondary shaft, synchronizing clutch, epicyclic mechanism, and the rest part of the gear box will work as a rigid body, as shown in Figure 3. In this case, the dynamic equation of the driveline could be described as:

$\left(I_{\mathrm{e}}+I_{\mathrm{mc}}+I_{\mathrm{s}}+I_{\mathrm{v}}\right) \dot{\omega}_{\mathrm{e}} \tau_{\mathrm{es}}=\frac{T_{\mathrm{m}}}{\tau_{\mathrm{epi}}}-\left(C_{\mathrm{e}}+C_{\mathrm{mc}}+C_{\mathrm{s}}+C_{\mathrm{v}}\right) \frac{\omega_{\mathrm{e}}}{\tau_{\mathrm{es}}}-\frac{T_{\mathrm{r}}}{\tau_{\mathrm{epi}} \tau_{\mathrm{c}}}$
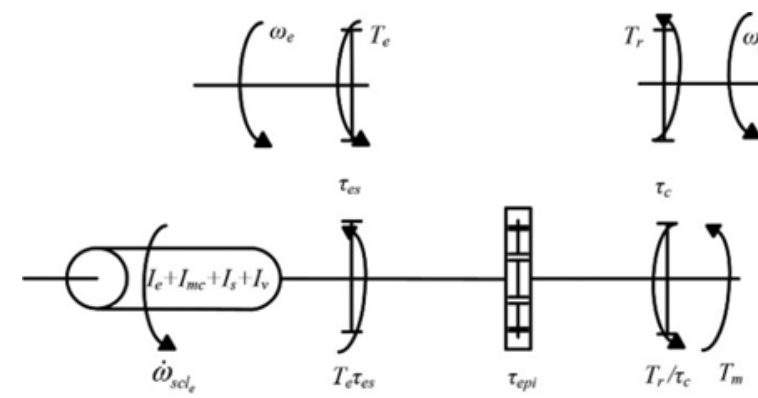

Figure 3. Free body diagram when synchronizing clutch engaged, gear disengaged.

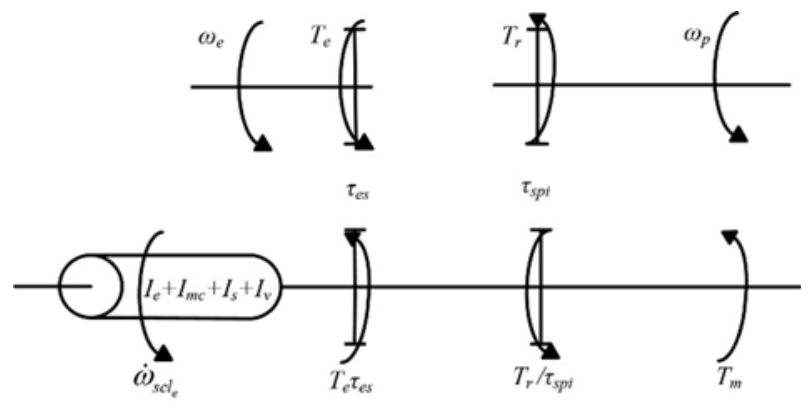

Figure 4. Free body diagram when synchronizing clutch engaged, gear disengaged.

In order to keep the transmission size small, the torque delivery compacity of the synchronizing clutch will be precisely designed to overcome the moment of inertia $\left(T_{\mathrm{e}}\right.$ is controlled to be zero) and other resistant force of the idling inner combustion engine and of the secondary shaft, which also implies that the synchronizing clutch is unable to directly transmit torque from engine to the primary shaft.

\subsection{Anticipated Gear Engaged, Synchronizing Clutch} Disengaged

After the synchronizing clutch locks up, the synchronizer dog teeth will immediately lock up the anticipated gear. Then the control unit simultaneously recovers the engine torque supply, cuts off the motor and discharges the synchronizing clutch. After finishing above operations, the proposed transmission will work as vehicle normal cruise.

The dynamics of the driveline, as shown in Figure 4, could be described as:

$$
\left(I_{\mathrm{e}}+I_{\mathrm{mc}}+I_{\mathrm{s}}+I_{\mathrm{v}}\right) \dot{\omega}_{\mathrm{e}} \tau_{\mathrm{es}}=T_{\mathrm{e}} \tau_{\mathrm{es}}-\left(C_{\mathrm{e}}+C_{\mathrm{mc}}+C_{\mathrm{s}}+C_{\mathrm{v}}\right) \frac{\omega_{\mathrm{e}}}{\tau_{\mathrm{es}}}-\frac{T_{\mathrm{r}}}{\tau_{\mathrm{spi}}}
$$

\section{CONTROL STRATEGY}

Since the state mentioned in Section 4.3 is the vehicle normally cruise, comparing the state mentioned in Section 4.1 with the one in Section 4.2, the only difference is that the latter one is not under the impact of synchronizing clutch friction torque, then only the former one is discussed for the sake of simplicity.

The control target of the state mentioned in Section 4.1. is concluded as: Ensure fast engagement and less torque fluctuation of the proposed synchronizing clutch; manipulate the torque complementary motor to prevent, or at least reduce the torque interruption as soon as possible.

\subsection{Synchronizing Clutch Control while Clutch Pads} Slipping

The dynamics of $\Delta \omega_{\text {scl }}$ could be described as the difference of the Equation (9) and the Equation (10). It holds: 


$$
\begin{aligned}
\Delta \dot{\omega}_{\mathrm{scl}}= & \dot{\omega}_{\mathrm{scl}_{\mathrm{e}}}-\dot{\omega}_{\mathrm{scl}_{\mathrm{p}}}=c_{1} T_{\mathrm{e}}-\left(c_{2}-c_{4}\right) \omega_{\mathrm{scl}_{\mathrm{e}}}-c_{3} T_{\mathrm{scl}}-c_{4} \Delta \omega_{\mathrm{scl}} \\
& -\frac{T_{\mathrm{m}}}{I_{\mathrm{v}} \tau_{\mathrm{epi}}}+\frac{T_{\mathrm{r}}}{I_{\mathrm{v}} \tau_{\mathrm{epi}} \tau_{\mathrm{c}}}
\end{aligned}
$$

where $c_{1}=\frac{\tau_{\mathrm{es}}}{\left(I_{\mathrm{e}}+I_{\mathrm{mc}}+I_{\mathrm{s}}\right)}$

$$
c_{2}=\frac{\left(C_{\mathrm{e}}+C_{\mathrm{mc}}+C_{\mathrm{s}}\right) \tau_{\mathrm{es}}^{2}}{\left(I_{\mathrm{e}}+I_{\mathrm{mc}}+I_{\mathrm{s}}\right)}
$$$$
c_{3}=\left(\frac{1}{I_{\mathrm{e}}+I_{\mathrm{mc}}+I_{\mathrm{s}}}+\frac{1}{I_{\mathrm{v}}}\right) \operatorname{sgn}\left(\Delta \omega_{\mathrm{scl}}\right)
$$$$
c_{4}=\frac{c_{\mathrm{v}} \tau_{\mathrm{epi}} \tau_{\mathrm{c}}}{I_{\mathrm{v}}}
$$

$T_{\mathrm{e}}, T_{\mathrm{r}}, \omega_{\text {scl }_{\mathrm{e}}}$ will be considered as the disturbance $\Gamma_{\mathrm{scl}}$ of the system. $T_{\mathrm{e}}$ could be estimated by throttle angle $\theta$ and engine output shaft speed $\omega_{\mathrm{e}}$, then be expressed as $\hat{T}_{\mathrm{e}}(\theta$, $\left.\omega_{e}\right) ; T_{\mathrm{r}}$ could be estimated by Equation (11) as $\hat{T}_{\mathrm{r}}\left(\omega_{\mathrm{p}}\right) ; \hat{\omega}_{\mathrm{scl}_{\mathrm{e}}}$ is proportional to the measurable variable $\omega_{\mathrm{e}}$ according to Equation (7). Then, the $\Gamma_{\text {scl }}$ could be estimated as:

$$
\Gamma_{\mathrm{scl}}=\hat{\Gamma}_{\mathrm{scl}}=c_{1} \hat{T}_{\mathrm{e}}-\left(c_{2}-c_{4}\right) \hat{\omega}_{\mathrm{scl}}+\frac{\hat{T}_{\mathrm{r}}}{I_{\mathrm{v}} \tau_{\mathrm{epi}} \tau_{\mathrm{c}}}
$$

To clearly observe the torque fluctuation while synchronizing clutch engaging, the control input $u_{1}, u_{2}$ are confirmed as $\dot{T}_{\mathrm{scl}}$ and $T_{\mathrm{m}}$. Then Equation (15) could be rewritten as:

$$
\Delta \dot{\omega}_{\mathrm{scl}}=-c_{4} \Delta \omega_{\mathrm{scl}}-c_{3} \int u_{1} d t-\frac{1}{\tau_{\mathrm{epi}}} \int u_{2} d t+\Gamma_{\mathrm{scl}}
$$

5.2. Torque Complementary Motor Control while the Synchronizing Clutch Pads Slipping

As analyzed in Equation (12), the dynamics of primary shaft while the synchronizing clutch pads slipping could be described as:

$\dot{\omega}_{\mathrm{p}}=\dot{\omega}_{\mathrm{scl}_{\mathrm{p}}} \tau_{\mathrm{epi}} \tau_{\mathrm{c}}=\frac{T_{\mathrm{m}} \tau_{\mathrm{c}}}{I_{\mathrm{v}}}+c_{5} T_{\mathrm{scl}}-c_{6} \omega_{\mathrm{p}}-\frac{T_{\mathrm{r}}}{I_{\mathrm{v}}}$

where $c_{5}=\frac{\operatorname{sgn}\left(\Delta \omega_{\mathrm{scl}}\right) \tau_{\mathrm{epi}} \tau_{\mathrm{c}}}{I_{\mathrm{v}}}$

$c_{6}=\frac{c_{\mathrm{v}} \tau_{\mathrm{epi}}^{2} \tau_{\mathrm{c}}^{2}}{I_{\mathrm{v}}}$

$T_{\mathrm{r}}$ is considered as disturbance $\Gamma_{\mathrm{m}}$ here. Control input $u_{1}$, $u_{2}$ are also $\dot{T}_{\text {scl }}$ and $\dot{T}_{\mathrm{m}}$. Then, it holds:

$\Gamma_{\mathrm{m}}=\hat{\Gamma}_{\mathrm{m}}=-\frac{\hat{T}_{\mathrm{r}}}{I_{\mathrm{v}}}$

The Equation (18) could be rewritten as:

$\dot{\omega}_{\mathrm{p}}=\int \frac{u_{2} \tau_{\mathrm{c}}}{I_{\mathrm{v}}} d t+c_{5} \int u_{1} d t-c_{6} \omega_{\mathrm{p}}+\Gamma_{\mathrm{m}}$

The output torque $T_{\text {out }}$ of the primary torque is proportional to measurable output $\dot{\omega}_{\mathrm{p}}$ as:
$T_{\text {out }}=I_{\mathrm{v}} \dot{\omega}_{\mathrm{p}}$

Then the problem of keeping the output torque as flat as possible could be converted to keep the dynamics of the angular velocity of the primary shaft $\dot{\omega}_{\mathrm{p}}$ as flat as possible.

In vector form, the control input signal and the output signal are given as:

$\boldsymbol{U}=\left[\begin{array}{l}\dot{T}_{\mathrm{scl}} \\ \dot{T}_{\mathrm{m}}\end{array}\right]$

$\boldsymbol{y}=\left[\begin{array}{c}\Delta \omega_{\mathrm{scl}} \\ \dot{\omega}_{\mathrm{p}}\end{array}\right]$

The modeled disturbance is described as:

$\Gamma=\left[\begin{array}{c}\Gamma_{\mathrm{scl}} \\ \Gamma_{\mathrm{m}}\end{array}\right]$

Taking Laplace transform of Equation (17) without $\Gamma_{\text {scl }}$ and Equation (20) without $\Gamma_{\mathrm{m}}$, the transfer function matrix $\boldsymbol{G}(s)$ from the two input signals to the two output signals are obtained as follows:

$$
\begin{aligned}
& \boldsymbol{Y}(s)=\boldsymbol{G}(s) \boldsymbol{U}(s) \\
& {\left[\begin{array}{c}
\Delta \omega_{\mathrm{scl}} \\
\dot{\omega}_{\mathrm{p}}
\end{array}\right]=\left[\begin{array}{ll}
-\frac{c_{3}}{s\left(s+c_{4}\right)} & \frac{1}{\tau_{\mathrm{epi}} s\left(s+c_{4}\right)} \\
\frac{c_{5}}{s\left(s+c_{6}\right)} & \frac{\tau_{\mathrm{c}}}{s\left(s+c_{6}\right) I_{\mathrm{v}}}
\end{array}\right]\left[\begin{array}{c}
\dot{T}_{\mathrm{scl}} \\
\dot{T}_{\mathrm{m}}
\end{array}\right]}
\end{aligned}
$$

The transfer function matrix $\boldsymbol{E}(\mathrm{s})$ from two disturbance signals $\Gamma_{\text {scl }}$ and $\Gamma_{\mathrm{m}}$ to the two output signals are described as:

$$
\begin{aligned}
& \boldsymbol{Y}(s)=\boldsymbol{E}(s) \Gamma(s) \\
& {\left[\begin{array}{c}
\Delta \omega_{\mathrm{scl}} \\
\dot{\omega}_{\mathrm{p}}
\end{array}\right]=\left[\begin{array}{cc}
\frac{1}{\left(s+c_{4}\right)} & 0 \\
0 & \frac{1}{\left(s+c_{6}\right)}
\end{array}\right]\left[\begin{array}{c}
\Gamma_{\mathrm{scl}} \\
\Gamma_{\mathrm{m}}
\end{array}\right]}
\end{aligned}
$$

The $\Delta \omega_{\mathrm{scl}}$ and $\dot{\omega}_{\mathrm{p}}$ are simultaneously influenced by $\dot{T}_{\mathrm{scl}}$ and $\dot{T}_{\mathrm{m}}$, which means the coupling of the $\dot{T}_{\mathrm{m}}$ on the $\Delta \omega_{\text {scl }}$ and of the $\dot{T}_{\text {scl }}$ on the $\dot{\omega}_{\mathrm{p}}$. The coupling effects are to be rejected, or reduced, as much as possible.

\section{CONTROLLER DESIGN}

\subsection{Control Objective and Its Nominal Model}

As mentioned above, the control objective could be described as manipulating $\dot{T}_{\text {scl }}$ and $\dot{T}_{\mathrm{m}}$ to make $\Delta \omega_{\text {scl }}$ and $\dot{\omega}_{\mathrm{p}}$ track their reference trajectory, which is called doublereference-tracking control in this article.

6.1.1. Reference trajectory of $\Delta \omega_{\text {scl }}$

Since we need to minimize the gearshift time to illustrate the effectiveness of the proposed structure, and the lockup of the synchronizing clutch tends to cause a sudden change 


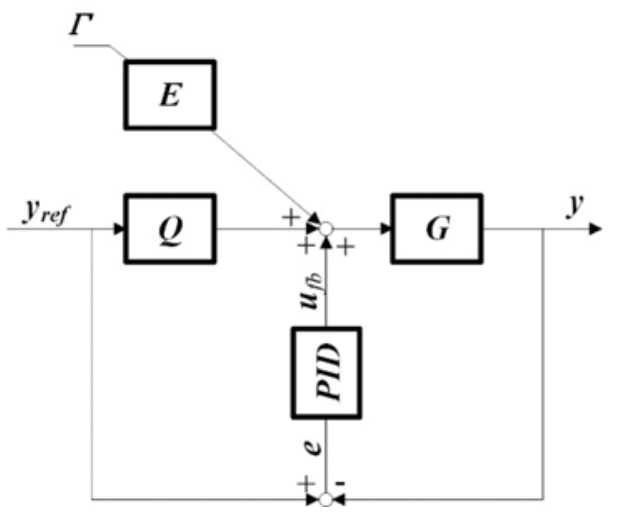

Figure 5. Controller diagram.

of the transmission output torque, the reference trajectory of $\Delta \omega_{\text {scl }}$ should satisfy:

(1) $\Delta \omega_{\text {scl }}$ decreases to zero as fast as possible within prespecified gearshift time $t_{\mathrm{f}}$.

(2) The change rate of the trajectory at $t_{\mathrm{f}}$ is zero.

The reference trajectory could be described by the polynomial below:

$\Delta \omega_{\mathrm{sclef}}=\frac{\left(t-t_{\mathrm{f}}\right)^{2}}{t_{\mathrm{f}}^{2}} \Delta \omega_{\mathrm{scl} 0}$

where $t_{\mathrm{f}}$ is the expected finish time; $\Delta \omega_{\text {sclo }}$ is the measured value of $\Delta \omega_{\text {scl }}$ while synchronizer disengaged.

\subsubsection{Reference trajectory of $\dot{\omega}_{\mathrm{p}}$}

In order to make driver feel consistent acceleration, the target value $\dot{\omega}_{\mathrm{p}_{\text {ref }}}$ should equal to the measured primary shaft rotational acceleration before the synchronizer dog teeth discharges $\dot{\omega}_{\mathrm{p} 0}$, then it holds:

$\dot{\omega}_{\mathrm{p}_{\mathrm{ref}}}=\dot{\omega}_{\mathrm{p} 0}$

Concluding Equations (29) and (30), it holds:

$\boldsymbol{y}_{\mathrm{ref}}=\left[\begin{array}{c}\Delta \omega_{\text {sclref }} \\ \dot{\omega}_{\mathrm{p}_{\mathrm{ref}}}\end{array}\right]$

\subsubsection{Description of control concept}

The double-reference-tracking control are simultaneously achieved by applying a decoupling controller $\mathrm{Q}$ as a feedforward controller, a feedback PID controller and a disturbance rejector E. Detailed design process will be described in Section 6.2.

\subsubsection{Nominal model}

Taking the values of Table A.1, the nominal transfer function matrix $\boldsymbol{G}_{0}$ and $\boldsymbol{E}_{\mathbf{0}}$ are defined as:

$$
\boldsymbol{G}_{0}(\boldsymbol{s})=\left[\begin{array}{cc}
-\frac{c_{3}}{s\left(s+c_{4}\right)} & \frac{1}{\tau_{\mathrm{epi}} s\left(s+c_{4}\right)} \\
\frac{c_{5}}{s\left(s+c_{6}\right)} & \frac{\tau_{\mathrm{c}}}{s\left(s+c_{6}\right) I_{\mathrm{v}}}
\end{array}\right]
$$

$$
\boldsymbol{E}_{0}(\boldsymbol{s})=\left[\begin{array}{cc}
\frac{1}{\left(s+c_{4}\right)} & 0 \\
0 & \frac{1}{\left(s+c_{6}\right)}
\end{array}\right]
$$

\subsection{Controller Design}

\subsubsection{Decoupling controller}

Decoupling control as demonstrated (Sanada et al., 2013) is basically feed-forward control where a decoupling controller is connected to the controlled object in series. The decoupling controller $\boldsymbol{Q}$ is determined such that the series connection of the controlled object $\boldsymbol{G}_{0}$ and the decoupling controller $\boldsymbol{Q}$ results in a diagonal matrix:

$\boldsymbol{G}_{\mathbf{0}} \boldsymbol{Q}=\left[\begin{array}{cc}T_{11}(s) & 0 \\ 0 & T_{22}(s)\end{array}\right]$

Multiplying inverse of the nominal model $\boldsymbol{G}_{\mathbf{0}}^{-1}$ to both sides, the decoupling controller $Q$ is solved:

$\boldsymbol{Q}=\boldsymbol{G}_{0}^{-1}\left[\begin{array}{cc}T_{11}(s) & 0 \\ 0 & T_{22}(s)\end{array}\right]$

The diagonal elements $T_{11}(s)$ and $T_{22}(s)$ should be determined so that each element of the matrix $Q(s)$ would become proper transfer function. In this case, transfer functions of first-order lag element are taken as:

$$
\begin{aligned}
& T_{11}(s)=\frac{\alpha}{s+\alpha} \\
& T_{22}(s)=\frac{\beta}{s+\beta}
\end{aligned}
$$

6.2.2. Feedback controller

The error signals $\boldsymbol{e}=\left[e_{\mathrm{scl}}, e_{\mathrm{m}}\right]^{\mathrm{T}}$ of the outputs are expressed as:

$\boldsymbol{e}=\boldsymbol{y}_{\mathrm{ref}}-\boldsymbol{y}$

The output of the proposed feedback controller $\boldsymbol{u}_{\mathrm{fb}}$ could be described as:

$\boldsymbol{u}_{\mathrm{fb}}=\boldsymbol{k}_{P} \boldsymbol{e}+\boldsymbol{k}_{\mathrm{I}} \int \boldsymbol{e} d t+\boldsymbol{k}_{\mathrm{D}} \frac{d \boldsymbol{e}}{d t}$

where $\boldsymbol{k}_{\mathrm{P}}=\left[K_{\mathrm{pscl}}, K_{\mathrm{pm}}\right]$

$\boldsymbol{k}_{\mathbf{I}}=\left[K_{\text {iscl }}, K_{\text {im }}\right]$

$\boldsymbol{k}_{\mathbf{D}}=\left[K_{\mathrm{dscl}}, K_{\mathrm{dm}}\right]$

In order to describe the feedback controller performance, an objective function $V$ is established as:

$$
V\left(K_{\mathrm{pscl}}, K_{\mathrm{iscl}}, K_{\mathrm{dscl}}, K_{\mathrm{pm}}, K_{\mathrm{im}}, K_{\mathrm{dm}}\right)=w_{\mathrm{scl}}\left\|\boldsymbol{e}_{\mathrm{scl}}\right\|_{1}+w_{\mathrm{m}}\left\|\boldsymbol{e}_{\mathrm{m}}\right\|_{1}
$$

where $\boldsymbol{e}_{\mathrm{scl}}$ and $\boldsymbol{e}_{\mathrm{m}}$ are respectively the time series of $\boldsymbol{e}_{\mathrm{scl}}$ and 
$e_{\mathrm{m}}$ during the simulation process. $w_{\mathrm{scl}}$ and $w_{\mathrm{m}}$ are weighting parameters.

The values of $w_{\text {scl }}=0.96$ and $w_{\mathrm{m}}=0.04$ are confirmed considering the priority of the proposed structure: to engage the synchronizing clutch in prespecified time.

The change of $V$ with respect to the six PID parameters could be observed by using MATLAB ${ }^{\circledR}$ command "sim", and then the PID parameters for different gears are tuned by using Nedler-Mead (Nedler and Mead, 1965) optimization control algorithm to find the minimum value of $V$. The PID parameters obtained for each gear up shift are listed in Table A.2. Detailed tuning process will be presented in a separate publication.

Introducing PID feedback controller into the system will contribute to reject the unmodeled disturbances, model error and unmodeled uncertainties, but the coupling effect will be deteriorated. To reduce such a side effect, a disturbance compensator is proposed. It's worth noting that the parameters are tuned with the presence of the disturbance compensator.

\subsubsection{Disturbance compensator}

The proposed disturbance compensator means to accomplish the two tasks listed below:

(1) Offset or reduce the negative effect to $\boldsymbol{y}$ caused by $\boldsymbol{\Gamma}$;

(2) Offset or reduce the coupling effect of $T_{\mathrm{m}}$ and $T_{\mathrm{scl}}$ caused by the PID feedback controller mentioned in Section 6.2.1

Then, disturbances for controller design are described as:

$$
\Gamma_{\mathrm{c}}(s)=\left[\begin{array}{c}
\Gamma_{\mathrm{sclc}} \\
\Gamma_{\mathrm{mc}}
\end{array}\right]
$$

where $\Gamma_{\text {sclc }}=c_{1} \hat{T}_{e}-\left(c_{2}-c_{4}\right) \hat{\omega}_{\text {scl }}-\frac{\hat{T}_{\mathrm{m}}}{\tau_{\mathrm{epi}}}+\frac{\hat{T}_{\mathrm{r}}}{\tau_{\mathrm{epi}} \tau_{\mathrm{c}}}$

$\Gamma_{\mathrm{mc}}=-\frac{\hat{T}_{\mathrm{r}}}{I_{\mathrm{v}}}+c_{5} \hat{T}_{\mathrm{scl}}$

$\hat{T}_{\mathrm{m}}$ and $\hat{T}_{\text {scl }}$ could be estimated as the output of torque observer.

The disturbance compensator is designed to satisfy the following equation as demonstrated (Sanada and Kitagawa, 1998):

$$
\boldsymbol{G}_{\mathbf{0}}(s) \boldsymbol{K}_{\mathbf{c d}} \Gamma_{\mathbf{c}}(s)+\boldsymbol{E}(s) \Gamma_{\mathbf{c}}(s)=\mathbf{0}
$$

And consequently:

$$
\boldsymbol{K}_{\mathrm{cd}}=-\boldsymbol{G}_{0}(s)^{-1} \boldsymbol{E}(s)
$$

\section{SIMULATION RESULT AND DISCUSSION}

For the proposed automated mechanical transmission, an upshift simulation model is made on commercial simulation software Simulink. Three system states are modeled by enabled subsystem. Three states respectively

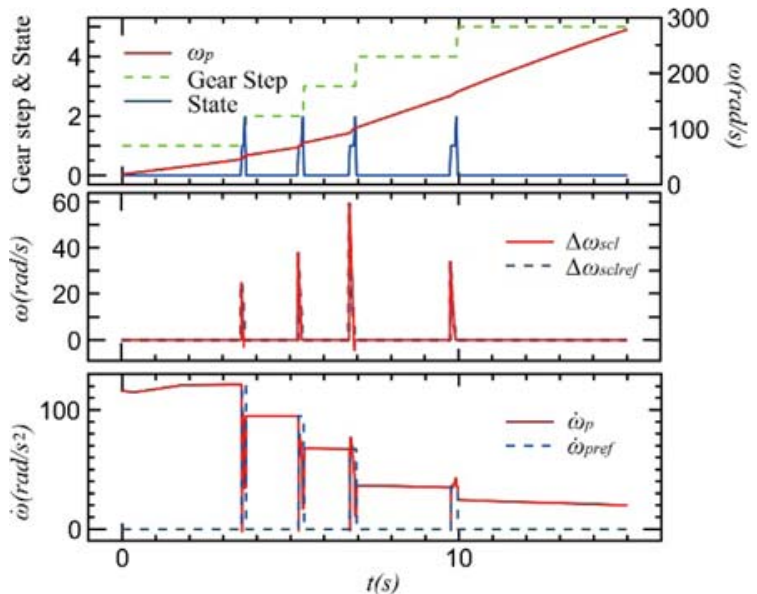

Figure 6. Simulation results of proposed controller while $\alpha$ $=10, \beta=200, \theta=0^{\circ}$.

present three driving circumstances from the 1st gear step to the 5th gear step as: synchronizing clutch disengaged, vehicle cruise on certain gear ratio (state 0 ); synchronizing clutch slipping, synchronizer disengage (state 1); synchronizing clutch engaged, synchronizer disengaged (state 2). Three system states make up a state loop in a numerical order to simulate the upshift operation, and the state transfer is achieved by Stateflow toolbox.

Simulation results of proposed controllers are shown in Figure 6. Gear step, state number, the angular velocity difference of synchronizing clutch pads, output speed and output acceleration are plotted in the figure.

When the system transfers from state 0 to state 1 , a sharp output acceleration decrease occurs. Such a sudden decrease is caused by a short torque interruption during the period after engine torque is cut off and before the motor provides sufficient complementary torque. The problem supposes to be deteriorated if the motor response is not fast enough. Gradually replacing engine torque with motor torque before system transfers state 0 to state 1 may fix such a problem.

As discussed in Section 6.2.2, the PID parameters are tuned by firstly considering engage the clutch (to ensure short shift time) to illustrate the effectiveness of the proposed structure. During gear upshift, the increase of $T_{\mathrm{m}}$ and $T_{\mathrm{scl}}$ simultaneously contributes to the decreasement of $\Delta \omega_{\mathrm{scl}}$ and the increasement of $\omega_{\mathrm{p}}$. Weighting parameters $w_{\mathrm{m}}$ could be adjusted larger for better output torque tracking performance in exchange of shorter gear shift time.

A dead zone block is applied to provide a target interval of $[-10,10]$ for $\Delta \omega_{\text {scl }}$ as the state transfer condition from state 1 to state 2 . If $\Delta \omega_{\text {scl }}$ is detected within the target interval, the system state will transfer. Such a setting simulates the synchronizing clutch lockup operation to ensure the synchronization of the primary shaft and the anticipated gear. The target interval could be adjusted larger in exchange of a shorter gear shift time and be 


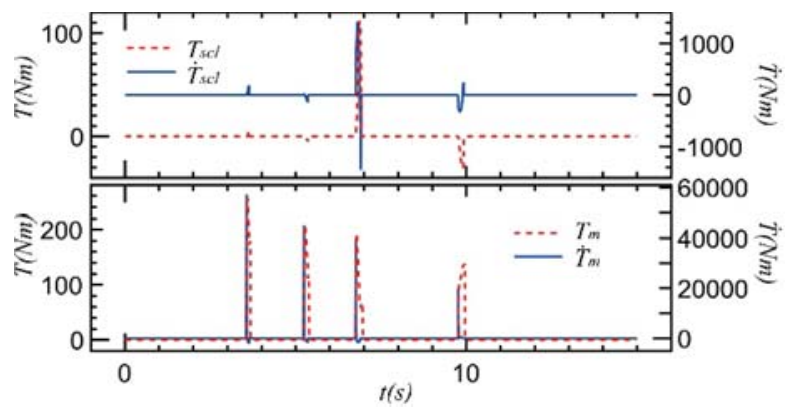

Figure 7. reference $\dot{T}_{\mathrm{m}}, \dot{T}_{\mathrm{scl}}$ and $T_{\mathrm{m}}, T_{\mathrm{scl}}$ while $\alpha=10, \beta=$ $200, \theta=0^{\circ}$.

adjusted smaller for less synchronizing clutch jerk. But if the target interval is too large, the engagement will be possible to cause huge torque fluctuation on the driveline, which may further damage the inner combustion engine. On the other hand, if the target interval is too small, the sensor may fail to detect the satisfied $\Delta \omega_{\text {scl }}$ within prespecified $0.2 \mathrm{~s}$.

The state 2 simulates the process that the synchronizer engages the anticipated gear for $0.1 \mathrm{~s}$. The whole system is driven by the motor as a rigid body during state 2 . While system transfers from state 2 back to state 0 , the reignition of the inner combustion engine may also cause driveline jerk, which may deteriorate the driving comfort.

From Figure 7, the reference values of $T_{\text {scl }}$ for state 1 and sate 2 in the upshift process from the 2 th to the 3 rd gear step and from the 4th to the 5th gear step are given as negative values to offset the output acceleration overshooting caused by decoupling controller, but according to physical theory, it's impossible for $T_{\text {scl }}$ to be negative while $\Delta \omega_{\mathrm{scl}}>0$, which indicates the system dynamics will be different in engineering application. It also worth noting that in the gear upshift process from the 3rd to the 4th gear step, to finish gearshift within prespecified time, the synchronizing clutch will cause severe driveline jerk.

As shown in Figure 8, to achieve such a system performance with the amount of engine power $P_{\mathrm{p}}$, the reference motor power is required to reach a maximum of over $40 \mathrm{~kW}$, which will cost more electrical energy from battery and more space in engine room. Detailed power and spin rate information are listed in Table 1. As torque interruption appears to be sharp in lower gear step, and quiet in higher ones, a $10 \mathrm{~kW}$ motor with $20 \mathrm{kw}$ maximum

Table 1. Motor peak reference power $P_{\mathrm{p}}(\mathrm{kW})$ and spin rate range $r_{\mathrm{m}}(\mathrm{rpm})$.

\begin{tabular}{ccccc}
\hline & 1st-2nd & 2nd-3rd & 3rd-4th & 4th-5th \\
\hline$P_{\mathrm{p}}$ & 24.25 & 28.54 & 36.03 & 40.89 \\
& & $1280 \sim$ & $1756 \sim$ & $3054 \sim$ \\
$r_{\mathrm{m}}$ & $862 \sim 994$ & 1428 & 1938 & 3168 \\
\hline
\end{tabular}

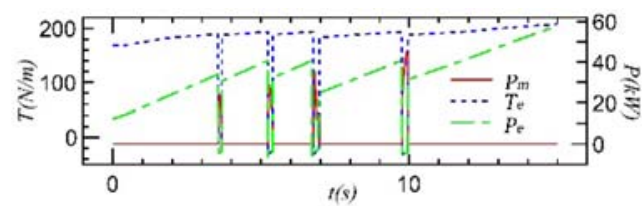

Figure 8. Engine torque and reference motor power.

Table 2. Friction losses in synchronizing clutch during gear upshift processes $W_{\text {syn }}$.

\begin{tabular}{lcccc}
\hline & 1st-2nd & 2nd-3rd & 3rd-4th & 4th-5th \\
\hline $\begin{array}{l}\text { Friction } \\
\text { loss }(\mathrm{J})\end{array}$ & 16.33 & 73.59 & 199.31 & 277.71 \\
\hline
\end{tabular}

power and 3,000 rpm maximum spin rate would be sufficient, even though the torque compensation may be compromised. By increasing the gear ratio of the complementary gear pair $\tau_{\mathrm{c}}$, the size of the electrical motor are expected to be smaller, but the value of $\tau_{\mathrm{c}}$ are highly corelated with the gear ratio of the epicyclic mechanism $\tau_{\text {epi }}$ and the gear ratio of the anticipated gear pair from secondary shaft to primary shaft $\tau_{\text {spi }}$ according to Equation (7). In addition, the epicyclic mechanism is too complicated, which will make increase $\tau_{\mathrm{c}}$ for lower electrical motor power, smaller motor size a conundrum to be solved in further research.

The friction losses of each gear upshift process are listed in Table 2. The relatively small friction loss swift gearshift will positively contribute to the fuel consumption reduction, which shows the cost penalty of such a complex mechanism.

Consider the possibility of applying the proposed automatic transmission on $\mathrm{EV}$, the proposed structure would be able to attain better driving comfort and be simplifier since the motor is more controllable in a wider angular velocity range compared with inner combustion engine.

\section{CONCLUSION}

The purpose of this paper is to show the proposed transmission's detailed analysis, effectiveness and possible problems in engineering application. Based on the mathematical model of the new structure, decoupling controller, feedback controller and disturbance compensator are consequently designed. The mathematical model with the designed controllers has been tested on Simulink. The results verify its effective swift gearshift. The defects need to be optimized and the possible problems may occur in engineering application have been discussed. Further research will be devoted to the optimization of the proposed structure and of the controllers to solve the existing defects, such as the complex gearshift assistant mechanism, the sharp torque 
decreasing after system state transfers from state 0 to state 1 , the huge driveline jerk during gear upshift from the $3 \mathrm{rd}$ to the 4th gear step, and minimize required motor power, etc. Detailed mathematical model of the synchronizing clutch and of the electrical motor will also be established since their nonlinear behavior will deteriorated their reference tracking performance. In addition, the proposed transmission will also be optimized in its robustness to the nonlinearity of the friction clutch, the electrical motor, the road inclination, the change of the tire diameter and the change of wind velocity, etc. In conclusion, the proposed structure is promising to achieve swift and smooth gearshift.

ACKNOWLEDGEMENT-This work is supported by the Collaborative Innovation Center of Major Machine Manufacturing in Liaoning, PR China, China Automobile Industry Innovation and Development Joint Fund (U1664257) and State Key Laboratory of Comprehensive Technology on Automobile Vibration and Noise \& Safety Control (W65-GNZX2018-0242).

\section{REFERENCES}

Dolcini, P., Wit, C. C. and Béchart, H. (2008). Lurch avoidance strategy and its implementation in AMT vehicles. Mechatronics 18, 5-6, 289-300.

Galvagno, E., Velardocchia, M. and Vigliani, A. (2009). A model for a flywheel automatic assisted manual transmission. Mechanism and Machine Theory 44, 6, 1294-1305.

Galvagno, E., Velardocchia, M. and Vigliani, A. (2011). Analysis and simulation of a torque assist automated manual transmission. Mechanical Systems and Signal Processing 25, 6, 1877-1886.

Gao, B., Chen, H., Lu, X. and Sanada, K. (2013a). Improved optimal controller for start-up of AMT trucks in consideration of driver's intention. Int. J. Automotive Technology 14, 2, 213-220.

Gao, B., Lei, Y., Ge, A., Chen, H. and Sanada, K. (2011a). Observer-based clutch disengagement control during gear shift process of automated manual transmission. Vehicle System Dynamics: Int. J. Vehicle Mechanics and Mobility 49, 5, 685-701.

Gao, B., Liang, Q., Xiang, Y., Guo, L. and Chen, H. (2015). Gear ratio optimization and shift control of 2-speed IAMT in electric vehicle. Mechanical Systems and Signal Processing, 50-51, 615-631.

Gao, B., Lu, X., Chen, H., Lu, X. and Li, J. (2013b). Dynamics and control of gear upshift in automated manual transmissions. Int. J. Vehicle Design 63, 1, 61-
83.

Gao, B., Lu, X., Li, J. and Chen, H. (2011b). Model predictive control of gear shift process in AMT trucks. Proc. IDETC2011, Washington, D.C, USA.

Lin, S., Chang, S. and Li, B. (2014). Gearshift control system development for direct-drive automated manual transmission based on a novel electromagnetic actuator. Mechatronics 24, 8, 1214-1222.

Lu, X., Chen, H., Wang, P. and Gao, B. (2011). Design of a data-driven predictive controller for start-up process of AMT vehicles. IEEE Trans. Neural Networks 22, 12, 2201-2212.

Maruyama, K., Kamiya, M. and Nishimaki, S. (2017). Current status and future prospects for each transmission model (in Japanese). Automotive Technology, Transmission Special Edition 71, 9, 12-17.

Nedler, J. A. and Mead, R. (1965). A simplex method for function minimization. The Computer Journal 7, 4, 308313.

Roozegar, M. and Angeles, J. (2018). A two-phase control algorithm for gear-shifting in a novel multi-speed transmission for electric vehicles. Mechanical Systems and Signal Processin, 104, 145-154.

Roozegar, M., Setiawan, Y. and Angeles, J. (2017). Design, modelling and estimation of a novel modular muti-speed transmission system for electric vehicle. Mechatronics, 45, 119-129.

Sanada, K. and Kitagawa, A. (1998). A study of twodegree-of-freedom control of rotating speed in an automatic transmission, considering modeling errors of a hydraulic system. Control Engineering Practice 6, 9, 1125-1132.

Sanada, K., Gao, B., Kado, N., Takamatsu, H. and Toriya, K. (2012). Design of a robust controller for shift control of an automatic transmission. Proc. Institution of Mechanical Engineers, Part D: J. Automobile Engineering 226, 12, 1577-1584.

Sanada, K., Kikuchi, K., Takamatsu, H. and Toriya, K. (2013). Decoupling control of torque and speed of automatic transmission. Proc. 12th Int. Symp. Fluid Control, Measurement and Visualization, Nara, Japan.

Shoji, M., Fukada, T., Shioiri, Y., Kato, K. and Ito, S. (2017). Development of suzuki AGS (in Japanese). Automotive Technology, Transmission Special Edition 71, 9, 21-26.

Sorniotti, A. (2009). Torque gap filler for automated manual transmissions: principles for the development of the control algorithm. SAE Paper No. 2009-01-0952.

Publisher's Note Springer Nature remains neutral with regard to jurisdictional claims in published maps and institutional affiliations. 


\section{APPENDIX}

Table A.1. Values of simulation model parameters.

\begin{tabular}{|c|c|c|c|c|c|}
\hline Parameters & Values & Unit & Parameters & Values & Unit \\
\hline$\tau_{\text {epi }}$ & {$\left[\begin{array}{lllll}1.0503 & 0.7640 & 0.4403 & 0.3333\end{array}\right]$} & - & $C_{\mathrm{v}}$ & 0.08 & $\mathrm{Nm}(\mathrm{rad} / \mathrm{s})$ \\
\hline$\tau_{\mathrm{es}}$ & 1.5 & - & $C_{\mathrm{s}}$ & 0.08 & $\mathrm{Nm}(\mathrm{rad} / \mathrm{s})$ \\
\hline$\tau_{\mathrm{c}}$ & 2 & - & $C_{\mathrm{e}}$ & 0.05 & $\mathrm{Nm}(\mathrm{rad} / \mathrm{s})$ \\
\hline$\tau_{\mathrm{df}}$ & 2 & - & $m$ & 1500 & $\mathrm{~kg}$ \\
\hline$t_{\mathrm{f}}$ & 0.2 & $\mathrm{~s}$ & $T_{\mathrm{w}}$ & 40 & $\mathrm{Nm}$ \\
\hline$I_{\mathrm{mc}}$ & 0.005 & $\mathrm{kgm}^{2}$ & $C_{\mathrm{A}}$ & 0.84 & - \\
\hline$I_{\mathrm{v}}$ & 5.08 & $\mathrm{kgm}^{2}$ & $R_{\mathrm{w}}$ & 0.2 & $\mathrm{~m}$ \\
\hline$I_{\mathrm{e}}$ & 0.2 & $\mathrm{kgm}^{2}$ & $\alpha$ & 10 & - \\
\hline$I_{\mathrm{s}}$ & 0.08 & $\mathrm{kgm}^{2}$ & $\beta$ & 200 & - \\
\hline$C_{\mathrm{mc}}$ & 0.2 & $\mathrm{Nm}(\mathrm{rad} / \mathrm{s})$ & $w_{\mathrm{c}}$ & 0.96 & - \\
\hline$C_{\mathrm{v}}$ & 0.08 & $\mathrm{Nm}(\mathrm{rad} / \mathrm{s})$ & $w_{\mathrm{m}}$ & 0.04 & - \\
\hline
\end{tabular}

Table A.2. PID parameters in different gear step.

\begin{tabular}{ccccccc}
\hline Gear step & $K_{\mathrm{pc}}$ & $K_{\mathrm{ic}}$ & $K_{\mathrm{dc}}$ & $K_{\mathrm{pm}}$ & $K_{\mathrm{im}}$ & $K_{\mathrm{dm}}$ \\
\hline 1st-2nd & 13.2519 & 32.9839 & -5.8639 & -24.1982 & 16.6443 & -0.6745 \\
2nd-3rd & -18.4216 & 43.8181 & -4.1748 & -19.9619 & 21.4796 & 10.4395 \\
3rd-4th & -166.9288 & 33.5740 & 29.5963 & 69.3260 & 118.3064 & -24.4907 \\
4th-5th & 139.9003 & 7.7468 & -142.9986 & 80.2402 & -95.1263 & 20.1743 \\
\hline
\end{tabular}

